\title{
Imaginary tune split and repulsion single-bunch instability mechanism in the presence of a resistive transverse damper and its mitigation
}

\author{
E. Métral『* \\ CERN, 1211 Geneva, Switzerland
}

(Received 22 January 2021; accepted 5 April 2021; published 21 April 2021)

\begin{abstract}
A resistive transverse damper is often needed in particle accelerators operating with many bunches and it is usually very efficient as it can considerably reduce the necessary amount of nonlinearities needed to reach beam stability through Landau damping. In the CERN LHC for instance, the required current in the Landau octupoles is predicted to be reduced by an order of magnitude for zero chromaticity (for the beam and machine parameters used during the last year of Run 2, in 2018, this corresponded to 2000 A without damper and 200 A with damper, knowing that the maximum current available in the Landau octupoles is $\sim 550$ A). However, a resistive transverse damper also destabilizes the single-bunch motion below the transverse mode coupling instability intensity threshold (for zero chromaticity), introducing a new kind of instability, which has been called ITSR instability (for imaginary tune split and repulsion). Until now, only one type of impedance-driven transverse coherent instability has been explained for a single bunch in a circular particle accelerator, at zero chromaticity and without a multiturn wake: the transverse mode coupling instability. A transverse mode coupling instability can also be observed in the presence of Landau damping, beam-beam, electron cloud or space charge. However, the ITSR instability exhibits a different mechanism, which is not due to mode coupling. The purpose of this article is to explain in detail both this new instability mechanism and its mitigation using a simplified analytical model, which has been carefully benchmarked, using the PyHEADTAIL macroparticle tracking code, by Oeftiger (one of the code's developers).
\end{abstract}

DOI: 10.1103/PhysRevAccelBeams.24.041003

\section{INTRODUCTION AND MOTIVATION}

A resistive transverse damper (TD) is needed, for instance, for multi-bunch operation in a machine like the CERN Large hadron collider (LHC) [1,2,3] and it has been working very well over the past decade, helping to reach twice the design peak luminosity. However, several simulations performed in the past with different (Vlasov solver and tracking) codes, considering a single bunch with zero chromaticity, revealed a more critical situation (as concerns the instability growth-rate or the required Landau octupole current needed to stabilize the beam) with TD than without [4,3] (and references therein). In 1998, Berg performed a theoretical analysis on a head-tail mode instability caused by a feedback, demonstrating that this instability is a general feature of machines with a transverse lowfrequency feedback [5]. The paper was kept very general, mentioning in conclusion that "the perturbation theory

\section{*Elias.Metral@cern.ch}

Published by the American Physical Society under the terms of the Creative Commons Attribution 4.0 International license. Further distribution of this work must maintain attribution to the author(s) and the published article's title, journal citation, and DOI. arguments are very general, and apply to longitudinal as well as transverse impedances and feedback": no pictures were shown and the possible mitigation methods were not discussed. In 2005, Karliner and Popov proposed to use a feedback to cure the transverse mode coupling instability (TMCI), and a destabilizing effect of the TD could be also observed on some pictures but no model/explanation describing the cause/mechanism of this instability was given: it was referred to as a sort of TMCI [6]. Furthermore, as in the previous reference, the possible mitigation methods were not mentioned.

It is interesting to observe that the two main mitigation methods which are used to stabilize transverse coherent instabilities, i.e., Landau damping and transverse dampers [1], can be detrimental for the single-bunch stability when mode coupling is involved. The destabilizing effect of Landau damping on TMCI was demonstrated for the first time by Chin in 1985 [7]. It was rediscussed recently in [3] using a simplified model where only the lowest two modes couple and it is currently under detailed analysis without performing any approximation [8]. The aim of this publication is to explain in detail the physical mechanism behind the instability induced by a resistive TD and how it can be mitigated.

As mentioned in Ref. [9], better characterizing an instability is the first step before trying to find appropriate 


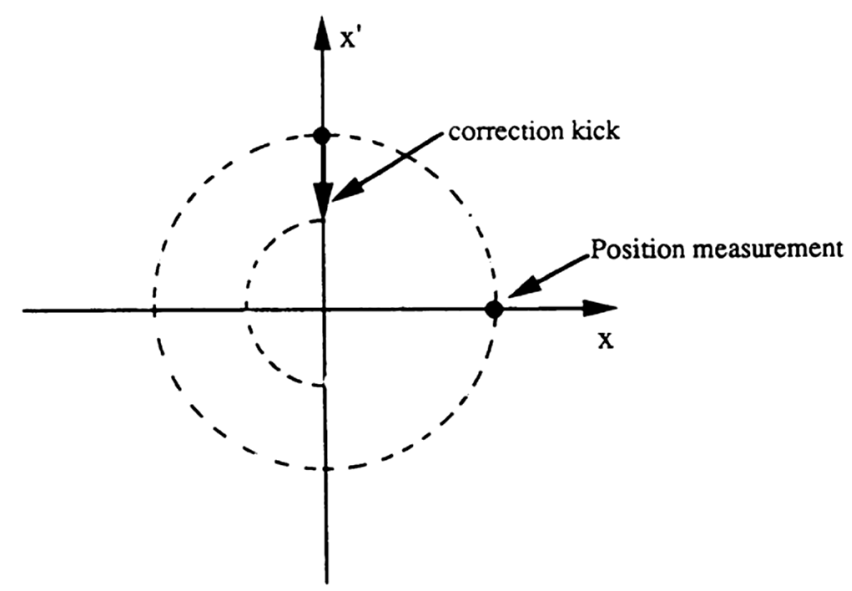

FIG. 1. Schematic picture (in the horizontal phase space) of the action of a conventional TD, which damps the center-of-charge motion of the beam. The position of the center-of-charge is measured by a pick-up and a correction kick is applied by a kicker with a betatron phase advance with respect to the pick-up of $90^{\circ}$. mitigation measures and push the performance of a particle accelerator. The ITSR instability is an additional instability mechanism which should be added to the list of the other transverse coherent instabilities already explained in the past: (i) the head-tail instability (for nonzero chromaticity), which is usually mitigated with Landau damping (for which the notion of stability diagram is valid when all the modes can be treated independently) [1]; (ii) the TMCI (for zero chromaticity), which is usually not mitigated with Landau damping as it requires a huge tune spread (of the order of the synchrotron tune, which might lead to other issues) [1]. Note that, as mentioned previously, Landau damping can be detrimental to the TMCI [7], as well as the detuning impedance (in asymmetric structures) [10], and that a TMCI can also be observed with beam-beam [11], electron cloud [10] or space charge [12]; and (iii) the family of instabilities due to loss of Landau damping, such as from linear coupling [13], from beam-beam [14] or from noise [15]. It is worth mentioning that a new kind of instability was also revealed recently for coasting beams in the presence of detuning impedance, where the most critical
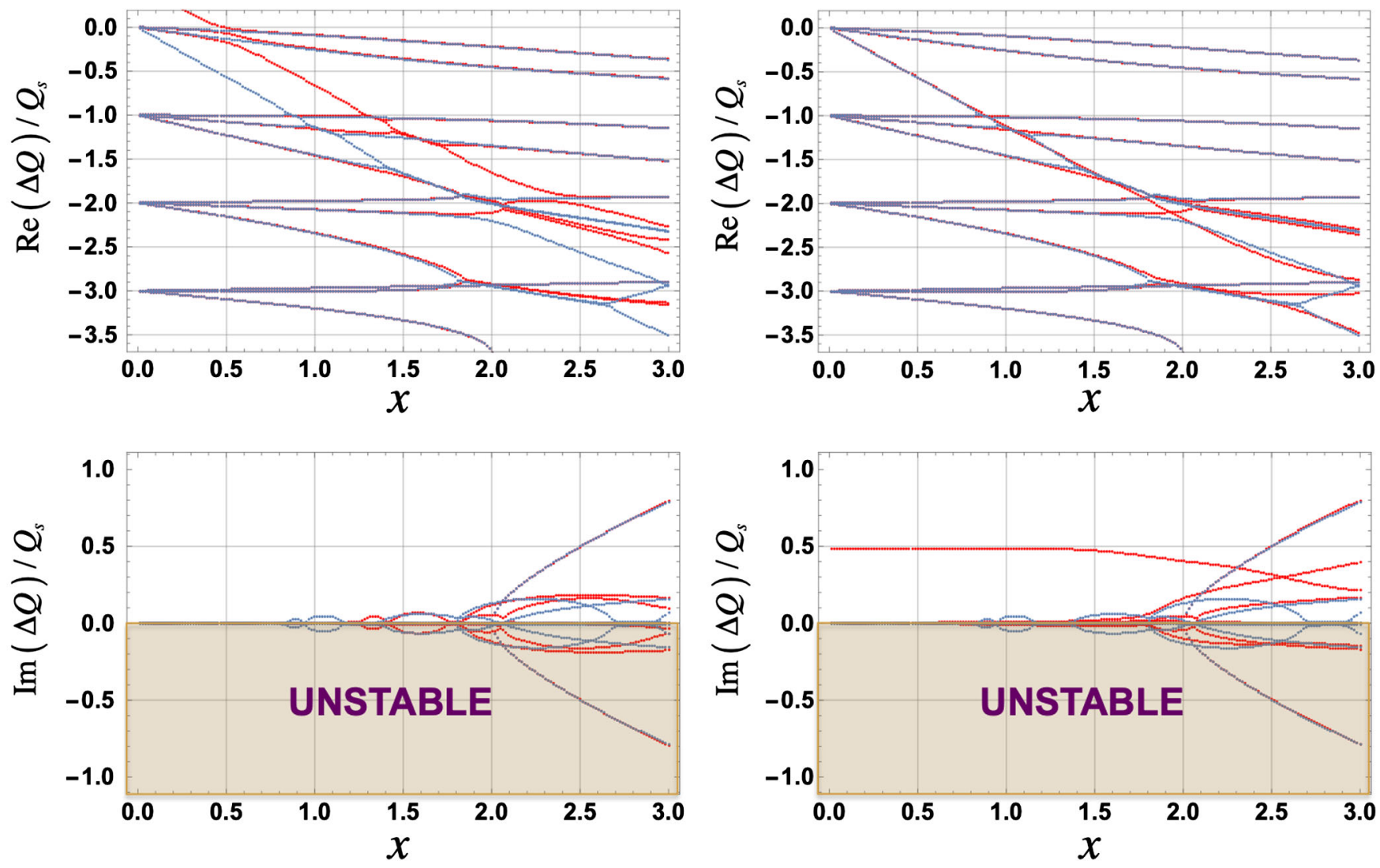

FIG. 2. Usual TMCI plots (showing the evolution of the real and imaginary parts of the complex tune shift solution of the eigenvalue problem, normalized by the synchrotron tune $Q_{s}$, vs $x$ a normalized parameter proportional to the bunch intensity [4]) from GALACTIC for the case of a bunch with a longitudinal "water-bag" distribution [19] interacting with a broad-band resonator impedance with a quality factor of 1 and a resonance frequency $f_{r}$ such that $f_{r} \tau_{b}=2.8$ (long-bunch regime, with $\tau_{b}$ the full, 4-sigma, bunch length). This approximately describes the CERN super proton synchrotron (SPS) case. Left: without TD in blue and with a reactive TD in red $\left(\Delta Q_{T D} / Q_{s}=0.48\right)$; Right: without TD in blue and with a resistive TD in red $\left(\Delta Q_{T D} / Q_{s}=0.48 j\right)$. 
instability changes plane above a certain intensity, and which could be explained by the coupling between adjacent fast and slow waves, so far not investigated in the literature [16].

This study was motivated by three questions, which were raised during the first two runs of the LHC: (1) why a chromaticity close to zero seemed to require a higher Landau octupole current than predicted during Run 1 (in 2011 and 2012) and during Run 2 (in 2015)? [17]; (2) why some past studies with a chromaticity close to zero revealed an instability with the TD, which is absent without TD? $[5,6,4,3]$ (and references therein); and (3) what should be the minimum operational chromaticity in the future in the LHC and High-Luminosity LHC? The GALACTIC Vlasov solver was developed few years ago $[4,3,18]$ to try and shed some light on these questions. Thanks to it, it was possible to explain the exact predicted instability mechanism in the presence of a resistive TD and to analyze in detail the effects of the gain and phase of the TD as well as the effect of Landau octupoles (providing Landau damping).

In this paper, the TD is assumed to act bunch by bunch, kicking the bunch as a whole and generating the following complex tune shift (with $j$ the imaginary unit)

$$
\Delta Q_{\mathrm{TD}}=\frac{e^{j \phi}}{2 \pi d},
$$
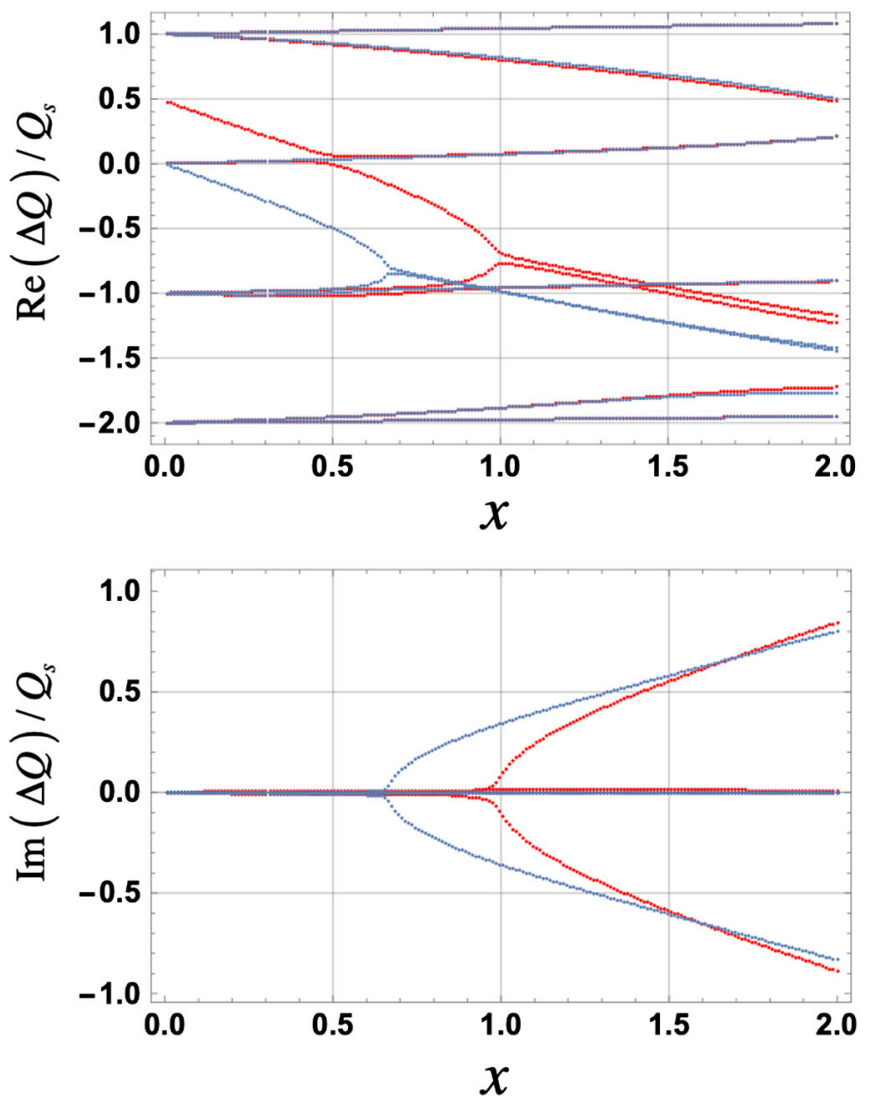

where $\phi$ is the betatron phase advance between the pick-up and the kicker, and $d$ is the TD damping time in machine turns (equal to $2 / G$ with $G$ the gain of the TD). If $\phi=90^{\circ}$, the TD is called resistive: it is a conventional damper/ feedback system, which damps the centre-of-charge motion of the beam (see Fig. 1). If $\phi=0^{\circ}$, the TD is called reactive: in this case, mode 0 is shifted up, which can raise the intensity threshold in the presence of TMCI between modes 0 and -1 (see below) [1].

\section{THE ITSR INSTABILITY MECHANISM FOR ZERO CHROMATICITY}

Starting from the linearized Vlasov equation and using a basis of the low-intensity eigenvectors of the problem, as proposed by Laclare and Garnier [19,20], the effect of a TD was added and a new Vlasov solver code was developed, called GALACTIC (for Garnier-Laclare coherent transverse instabilities code) [4], where an eigenvalue system needs to be solved. The GALACTIC Vlasov solver has been explained in $[4,18]$, where it was also successfully benchmarked against the PyHEADTAIL macroparticle tracking code [21] without TD.

While studying the effect of a TD on TMCI, it is important to distinguish between the long-bunch and short-bunch regimes as the impact of the TD is very different for the two regimes. In the long-bunch regime (see Fig. 2), the main
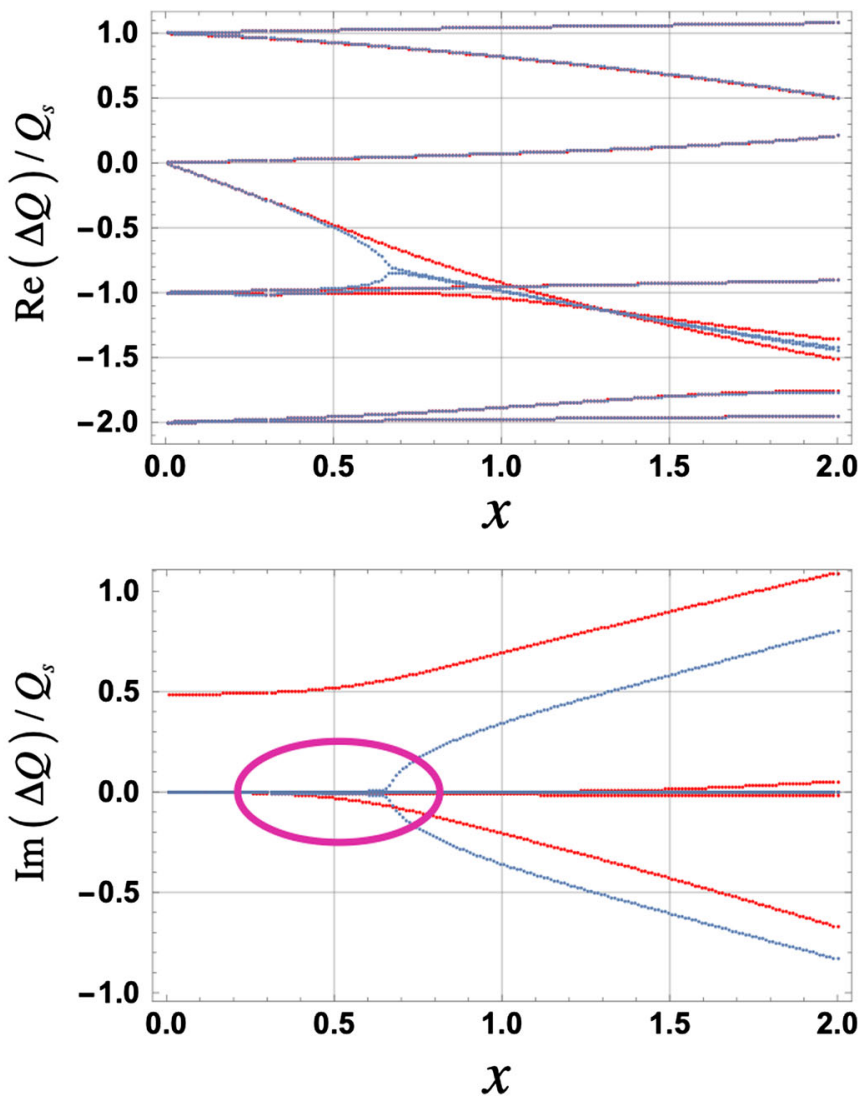

FIG. 3. Same as Fig. 2 but with $f_{r} \tau_{b}=0.8$ (short-bunch regime). This approximately describes the CERN LHC case. 
mode coupling takes place between high-order modes and the TD will not be able to modify it whatever its gain or phase. This is not the case for the short-bunch regime (see Fig. 3), for which the main mode coupling takes place between the modes 0 and -1 . In this case, a reactive TD is beneficial as it increases the TMCI intensity threshold, modifying the shift of mode 0 and pushing the mode coupling toward higher bunch intensities (see Fig. 3 left). A resistive TD, on the other hand, is detrimental as it decreases the intensity threshold (see Fig. 3 right). The exact mechanism will be explained below using a simplified model with only two modes as all the physics can be captured like this.

The matrix, which needs to be diagonalized in GALACTIC in this latter case, can be reasonably well approximated (for the purpose of the current study and over the relevant intensity range defined by the normalized parameter $x$ proportional to the bunch intensity) by this 2 by 2 matrix (taking into account only the modes 0 and -1 )

$$
H=\left[\begin{array}{cc}
-1 & -0.23 j x \\
-0.55 j x & -0.92 x+\Delta Q_{T D} / Q_{s}
\end{array}\right],
$$

where the term $\Delta Q_{T D} / Q_{s}$ is the contribution from the TD. The mode -1 is described by the top-left term while the mode 0 is described by the bottom-right one (the mode coupling terms being the off-diagonal ones). Note that this matrix was obtained from numerical computations with the GALACTIC Vlasov solver, and that it can take several forms: this explains why the off-diagonal terms are complex here, instead of being real, but this leads to the same results as it is the product between the two which matters. Figure 4 depicts the evolution of the eigenvalues for both cases with and without the TD and it can be observed that similar results as in Fig. 3 right are obtained. It is found indeed that introducing a resistive TD lowers the intensity threshold. In fact, it completely changes the nature of the instability as no intensity threshold is observed anymore (as already spotted in Refs. [5] and [6]): the bunch is unstable whatever the intensity (in the absence of Landau damping). Without $\mathrm{TD}$, an instability appears as a consequence of the coupling between two modes ( 0 and -1$)$. In the presence of the resistive TD, the mode coupling is suppressed but the interaction between the modes 0 and -1 in the presence of the TD pushes apart the imaginary parts and as the imaginary part of the mode -1 is 0 , it becomes negative, driving the mode -1 unstable.

The fact that the TD term in Eq. (2) is given by $\Delta Q_{T D} / Q_{s}$ explains why a TD is not very effective for machines with a large synchrotron tune $Q_{s}$. Indeed, assuming for instance a synchrotron tune as high as $Q_{s}=0.1$, a resistive TD with a damping time $d=50$ turns would almost not modify the TMCI picture, as can be seen in Fig. 5. In Fig. 6, a scan of the resistive TD strength
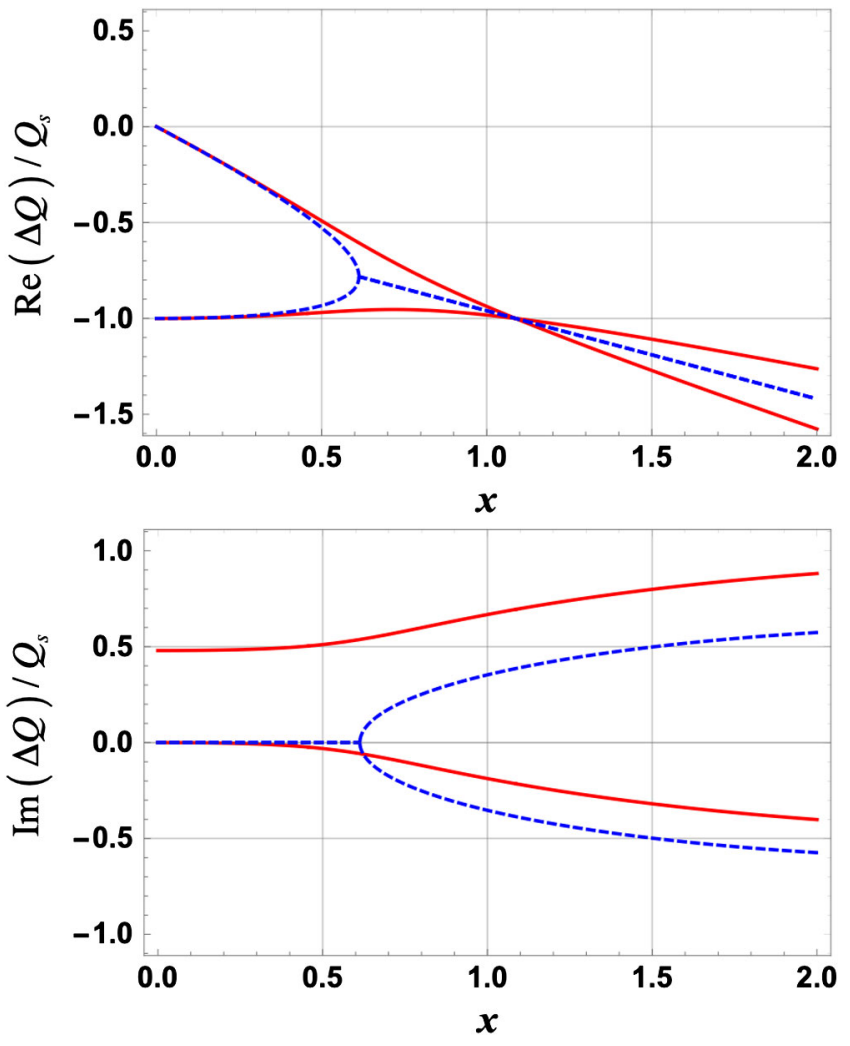

FIG. 4. Solutions of the diagonalization of the 2 by 2 matrix of Eq. (2) vs $x$ a normalized parameter proportional to the bunch intensity [4]: without (dotted blue line) and with (red line) the resistive $\operatorname{TD}\left(\Delta Q_{T D} / Q_{s}=0.48 j\right)$.

is performed from 0 to $0.98 j$, which reveals clearly its impact on the real and imaginary parts of the complex tune shift: the two modes always cross each other at the same intensity value, which is a bit less than two times the one without TD, and the imaginary part of the complex tune shift flattens with higher/similar (absolute) values below the TMCI intensity threshold without TD and smaller (absolute) values above the TMCI intensity threshold without TD.

As it was seen before that a reactive TD helps to increase the TMCI intensity threshold for the short-bunch regime (see Fig. 3 left), a first possibility to try and mitigate the ITSR instability is to add also a reactive part to the purely resistive TD (which is needed for multibunch operation). The case where the reactive part is equal to the resistive part is depicted in Fig. 7, where it can be clearly seen how the complex tune shit can be modified. It is seen in particular that the reactive part of the TD helps to reduce the (absolute) value of the imaginary part of the complex tune shift (below a certain intensity), i.e., it helps to increase the instability rise-time, but this is not sufficient to stabilize the bunch and Landau damping is required to mitigate this instability, as it will be explained in the next section. 

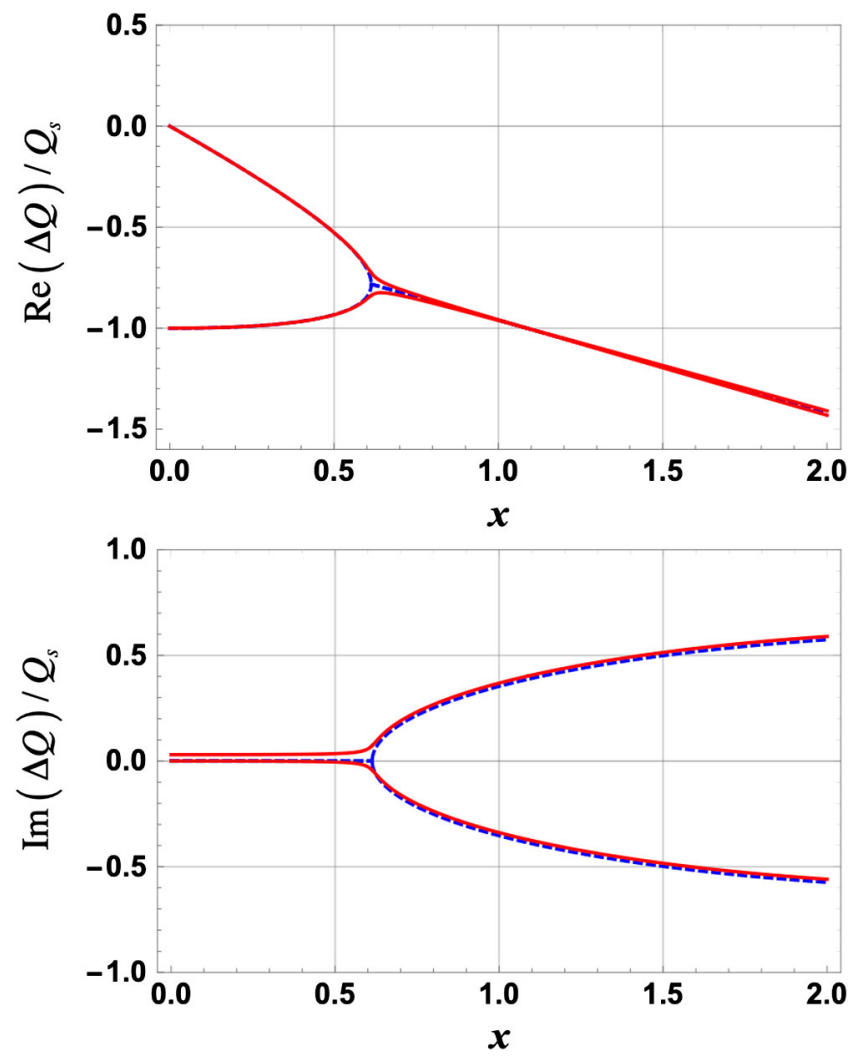

FIG. 5. Solutions of the diagonalization of the 2 by 2 matrix of Eq. (2) vs $x$ a normalized parameter proportional to the bunch intensity [4]: without (dotted blue line) and with (red line) the resistive TD assuming $Q_{s}=0.1$ and $d=50$ turns.

\section{MITIGATION OF THE ITSR INSTABILITY BY LANDAU DAMPING}

The ITSR instability mechanism involves several modes and therefore it cannot be studied using the usual Landau damping approach with a stability diagram, which assumes independent head-tail modes. In the general case, a nonlinear determinant needs to be solved, which is a quite challenging task without making some approximations $[7,8,22]$. As the ITSR instability mechanism described above involves the two modes 0 and -1 , the impact on Landau damping is studied by considering only these two modes and Eq. (3) needs to be solved [22]

$$
\left|\begin{array}{cc}
I_{m=-1}^{-1} & 0.23 j x \\
0.55 j x & I_{m=0}^{-1}+0.92 x-\Delta Q_{T D} / Q_{s}
\end{array}\right|=0
$$

where $I_{m}$ is the dispersion integral, which appears in the presence of a tune spread induced by the machine nonlinearities (controlled nonlinearities for transverse Landau damping are usually introduced thanks to octupoles, called Landau octupoles). I have solved Eq. (3) assuming an externally given elliptical tune spread, which leads to the circle stability diagram for the one-mode approach (i.e., assuming all the modes to be independent). In this case, the
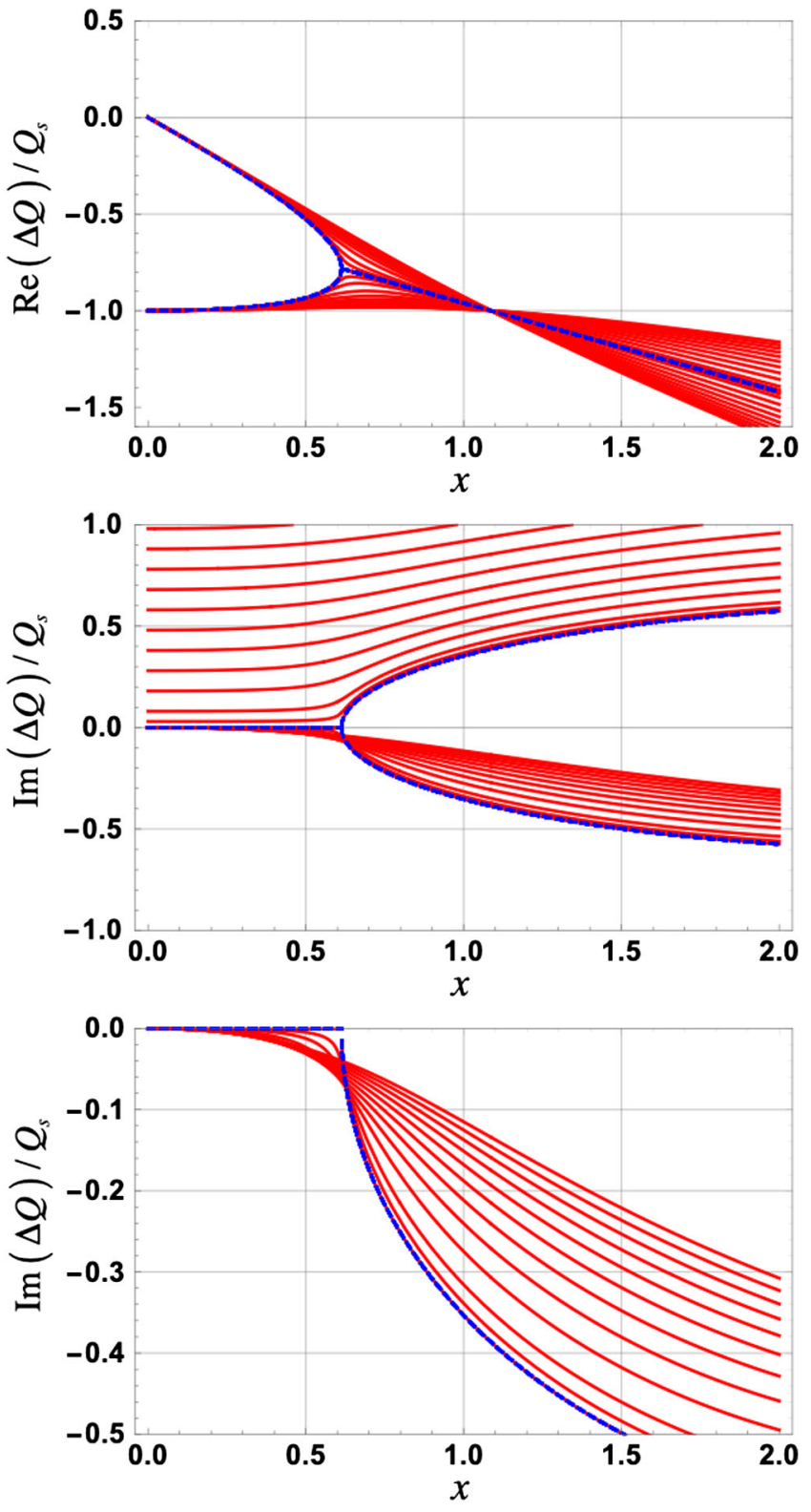

FIG. 6. Solutions of the diagonalisation of the 2 by 2 matrix of Eq. (2) vs $x$ a normalized parameter proportional to the bunch intensity [4]: without (dotted blue line) and with (red line) the resistive TD for $\Delta Q_{T D} / Q_{s}=0 ; 0.03 j ; 0.08 j ; 0.18 j ; 0.28 j$; $0.38 j ; 0.48 j ; 0.58 j ; 0.68 j ; 0.78 j ; 0.88 j ; 0.98 j$. The first two pictures describe the real and imaginary parts of the complex tune shift whereas the third one is a zoom of the imaginary part in the unstable region.

dispersion integral is given by [23] (with $y$ the unknown we are looking for)

$$
I_{m}=\frac{2}{y-m-j \sqrt{\Delta q^{2}-(y-m)^{2}}},
$$

where $\Delta_{q}$ is the tune spread (half width at the bottom of the distribution) normalised by the synchrotron tune $Q_{s}$. 

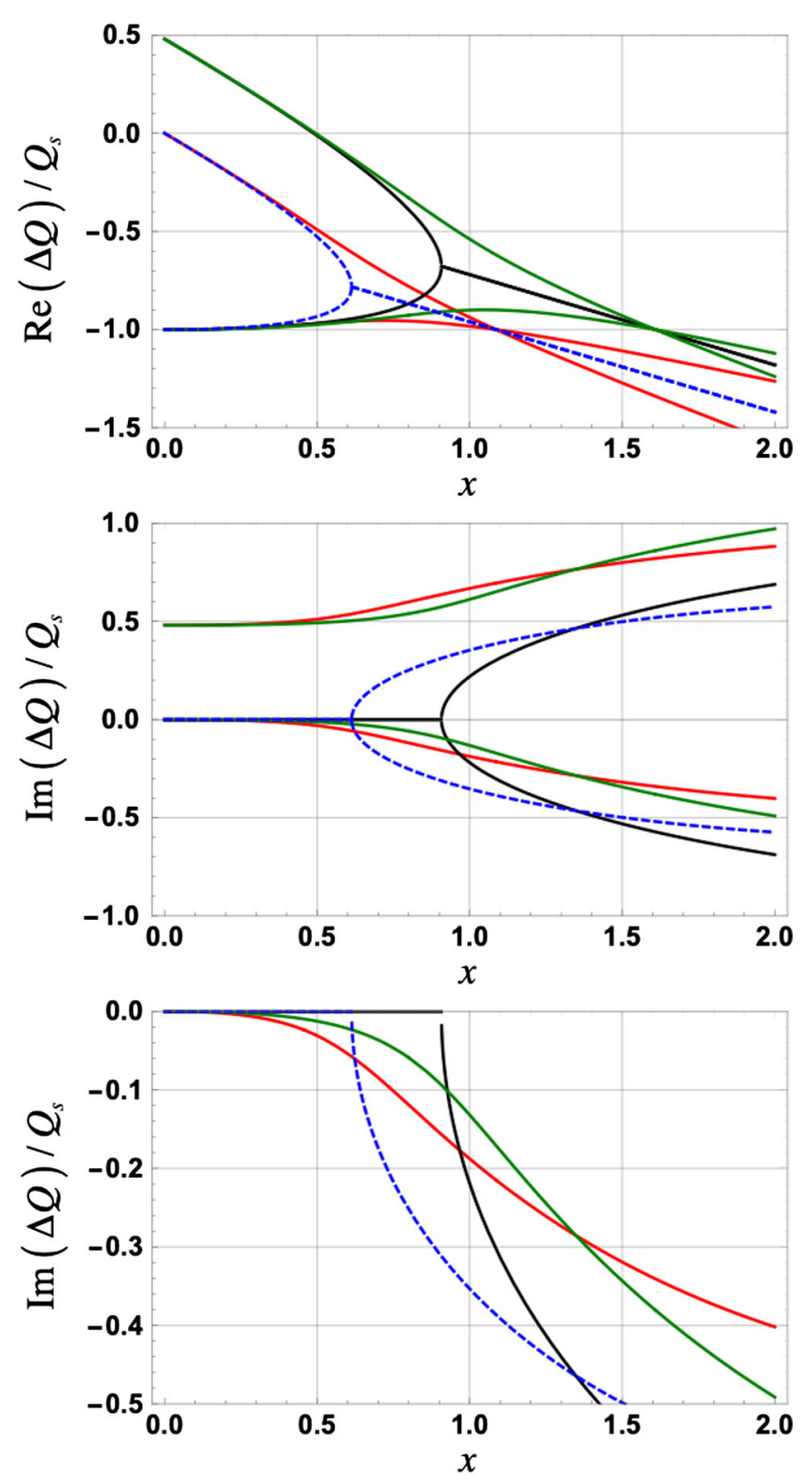

FIG. 7. Solutions of the diagonalization of the 2 by 2 matrix of Eq. (2) vs $x$ a normalized parameter proportional to the bunch intensity [4]: without TD (dotted blue line); with a resistive TD for which $\Delta Q_{T D} / Q_{s}=0.48 j$ (red line); with a reactive TD for which $\Delta Q_{T D} / Q_{s}=0.48$ (black line); and with both a resistive and reactive TD for which $\Delta Q_{T D} / Q_{s}=0.48(1+j)$ (green line). The first two pictures describe the real and imaginary parts of the complex tune shift whereas the third one is a zoom of the imaginary part in the unstable region.

The solution of Eq. (3), characterizing the two-mode approach, i.e., going beyond the notion of stability diagram, is compared to the one-mode approach in Fig. 8: it can be seen that below the TMCI intensity threshold (without TD), the one-mode approach (usual stability diagram) is fine, whereas above the TMCI intensity threshold (without TD), the two-mode approach is needed and (slightly) more tune spread is required. As the LHC has

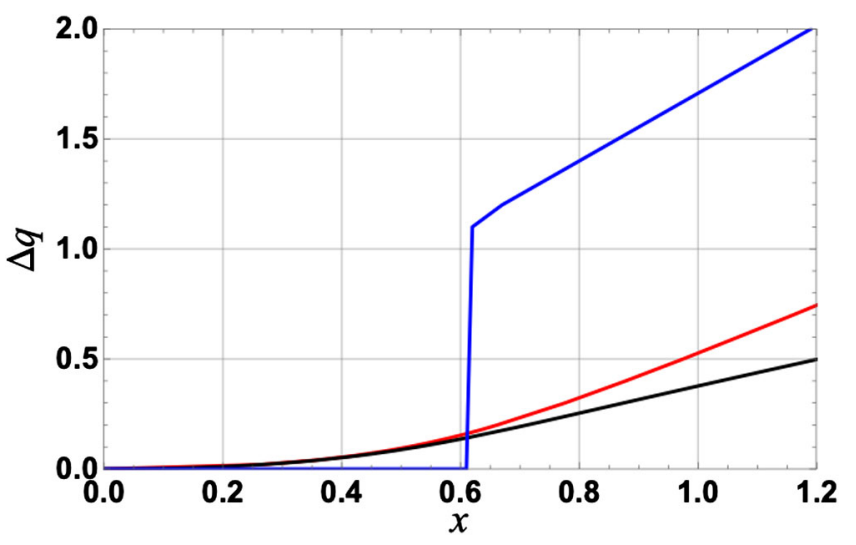

FIG. 8. Required tune spread (half width at the bottom of the assumed externally given elliptical tune distribution) normalized by the synchrotron tune $Q_{s}$ to reach bunch stability vs the normalised bunch intensity $x$ in the presence of TD for the case of Fig. 4: (black line) using the one-mode approach, leading to the usual stability diagram; (red line) with the two-mode approach from Eq. (3). The blue line corresponds to the case without TD (but considering the mode coupling between modes 0 and -1).

been operated until now below the TMCI intensity threshold (without TD), the one-mode approach used until now seems fully justified. It can also be concluded from Fig. 8 that a resistive TD has a detrimental effect below and a beneficial effect above the TMCI intensity threshold, as much less octupole current is needed for the latter case to reach beam stability through Landau damping than without a TD. This analytical description has been checked in detail through PyHEADTAIL macroparticle tracking simulations by Oeftiger, revealing a good agreement between the

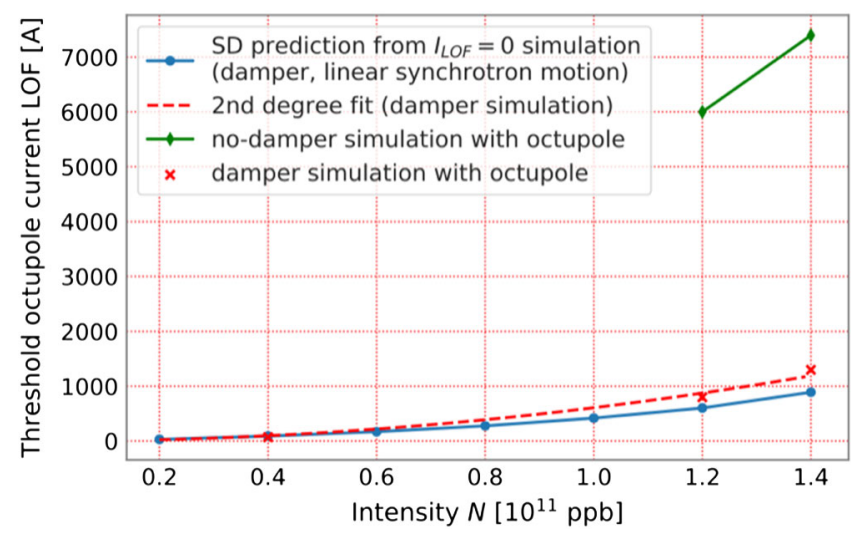

FIG. 9. Case of Fig. 8 analysed in detail through PyHEADTAIL tracking simulations (with Gaussian distributions), revealing a good agreement between the two approaches: (blue line) with the usual one-mode approach stability diagram (using the simulation results from PyHEADTAIL without Landau octupoles); (dotted red line) PyHEADTAIL simulations with both Landau octupoles and TD; (green line) PyHEADTAIL simulations with Landau octupoles but without TD. The TMCI intensity threshold without TD is at $\sim 0.8 \times 10^{11} \mathrm{ppb}$. This figure is courtesy of Oeftiger [24]. 
two approaches and confirming therefore that most of the physics has been well captured by the proposed model (see Fig. 9) [24].

\section{CONCLUSIONS}

A new single-bunch instability mechanism is revealed for zero chromaticity in the presence of a resistive transverse damper, which is clearly not a consequence of mode coupling (see Fig. 4). The explanation provided in this paper was confirmed by two other Vlasov solvers, DELPHI [25] (by Amorim, using a Gaussian longitudinal profile) and NHTVS [26] (by Antipov, using either a Gaussian or air-bag longitudinal profile), which could reproduce Figs. 3 and $4[3,4]$. The detailed instability mechanism could not be identified with PyHEADTAIL macroparticle tracking simulations only. However, the impact on Landau damping could be analysed in detail with PyHEADTAIL by Oeftiger and a good agreement was reached between the two approaches (compare Fig. 8 obtained with the analytical model presented in this paper and Fig. 9 obtained with PyHEADTAIL tracking simulations): in [24], Oeftiger concluded that "the proposed analytical model seems to explain all observations in full scale simulations very well". This confirmed the predicted detrimental effect of a resistive transverse damper below the TMCI intensity threshold and the beneficial effect of a resistive transverse damper above the TMCI intensity threshold. It can be concluded in particular that this ITSR instability can be stabilised by Landau damping: the required tune spread is the same as the one deduced from the usual one-mode approach stability diagram below the TMCI intensity threshold and it is a bit larger above the TMCI intensity threshold. It can also be concluded that this ITSR instability mechanism alone cannot explain why, in the CERN LHC, a chromaticity close to zero required a higher Landau octupole current than predicted (with the usual stability diagram approach) during Runs 1 and 2.

[1] A. W. Chao, Physics of Collective Beam Instabilities in High Energy Accelerators (John Wiley \& Sons, New York, 1993).

[2] W. Höfle, Progress in transverse feedbacks and related diagnostics for hadron machines, in 2013 International Particle Accelerator Conference (IPAC'13), Shanghai, China, 12-17 May 2013 (JACoW Publishing, Geneva, 2013), p. 3990-3994, paper FRXCA01, ISBN 978-395450-122-9.

[3] E. Métral, Destabilizing effect of resistive transverse dampers, in ICFA mini-Workshop on Mitigation of Coherent Beam Instabilities in Particle Accelerators, edited by E. Métral, T. Pieloni, and G. Rumolo (Zermatt, Switzerland, 2019), p. 221, https://doi.org/10.23732/CYRCP-2020-009.

[4] E. Métral, D. Amorim, S. Antipov, N. Biancacci, X. Buffat, and K. Li, Destabilising effect of the LHC transverse damper, in 9th International Particle Accelerator Conference (IPAC18), Vancouver, Canada, 2018 (JACoW Publishing, Geneva, 2018), page 3076, paper THPAF048.

[5] J. S. Berg, Head-tail mode instability caused by feedback, in Proceedings of the 6th European Particle Accelerator Conference, Stockholm, 1998 (IOP, London, 1998), pp. 942-944.

[6] M. Karliner and K. Popov, Theory of a feedback to cure transverse mode coupling instability, Nucl. Instrum. Methods Phys. Res., Sect. A 537, 481 (2005).

[7] Y.H. Chin, Hamiltonian formulation for transverse bunched beam instabilities in the presence of betatron tune spread, CERN Technical Report No. CERN SPS/85-9 (DI-MST), 1985.

[8] N. Mounet, Landau damping in the transverse plane, in ICFA mini-Workshop on Mitigation of Coherent Beam Instabilities in Particle Accelerators, edited by E. Métral, T. Pieloni, and G. Rumolo (Zermatt, Switzerland, 2019), p. 45 (https://doi.org/10.23732/CYRCP-2020-009).

[9] E. Métral, Intrabunch motion, Phys. Rev. Accel. Beams 24, 014401 (2021).

[10] G. Iadarola, L. Mether, N. Mounet, and L. Sabato, Linearized method for the study of transverse instabilities driven by electron clouds, Phys. Rev. Accel. Beams 23, 081002 (2020).

[11] S. White, X. Buffat, N. Mounet, and T. Pieloni, Transverse mode coupling instability of colliding beams, Phys. Rev. Accel. Beams 17, 041002 (2014).

[12] Y. Alexahin, Vlasov eigenfunction analysis of spacecharge and beam-beam effects, in ICFA mini-Workshop on Mitigation of Coherent Beam Instabilities in Particle Accelerators, edited by E. Métral, T. Pieloni, and G. Rumolo (Zermatt, Switzerland, 2019), p. 193, (https:// doi.org/10.23732/CYRCP-2020-009).

[13] L. R. Carver, X. Buffat, K. Li, E. Métral, and M. Schenk, Transverse beam instabilities in the presence of linear coupling in the large hadron collider, Phys. Rev. Accel. Beams 21, 044401 (2018).

[14] X. Buffat, W. Herr, N. Mounet, T. Pieloni, and S. White, Stability diagrams of colliding beams in the large hadron collider, Phys. Rev. Accel. Beams 17, 111002 (2014).

[15] S. V. Furuseth and X. Buffat, Loss of transverse Landau damping by noise and wakefield driven diffusion, Phys. Rev. Accel. Beams 23, 114401 (2020).

[16] N. Biancacci, E. Métral, and M. Migliorati, Fast-slow mode coupling instability for coasting beams in the presence of detuning impedance, Phys. Rev. Accel. Beams 23, 124402 (2020).

[17] E. Métral, G. Arduini, J. Barranco, N. Biancacci, X. Buffat, L. R. Carver, G. Iadarola, K. Li, T. Pieloni, A. Romano, G. Rumolo, B. Salvant, M. Schenk, and C. Tambasco, Measurements and interpretation of transverse beam instabilities in the CERN Large Hadron Collider (LHC) and extrapolations to HL-LHC, in 57th ICFA Advanced Beam Dynamics Workshop on High-Intensity and High-Brightness Hadron Beams: Malmö, Sweden, 3-8 July 2016 (JACoW Publishing, Geneva, 2016), pp. 254-259 paper TUAM2X01, ISBN 9783-95450-178-6. 
[18] E. Métral and M. Migliorati, Longitudinal and transverse mode coupling instability: Vlasov solvers and tracking codes, Phys. Rev. Accel. Beams 23, 071001 (2020).

[19] J. L. Laclare, Bunched beam coherent instabilities, CERN Technical Report CERN-1987-003-V-1, 1987.

[20] J. P. Garnier, Instabilités cohérentes dans les accélérateurs circulaires, Ph.D. thesis, Institut National Polytechnique de Grenoble, Grenoble, France, 1987.

[21] PyHEADTAIL. https://github.com/PyCOMPLETE/ PyHEADTAIL.

[22] R. Cappi, E. Métral, and D. Möhl, Transverse coherent instabilities in the presence of linear coupling, in Proceedings of the European Particle Accelerator Conference, Vienna, 2000 (EPS, Geneva, 2000), pp. 1155-1157, paper WEP4A08.
[23] E. Métral, Theory of coupled Landau damping, Part. Accel. 62, 259 (1998), https://s3.cern.ch/inspire-prod-files-0/ 00fe7df431d5072163a27345e2479501.

[24] A. Oeftiger, Stability around $Q^{\prime}=0$ and the LHC damper instability, 146th HiLumi WP2 meeting, CERN, Geneva, Switzerland, https://indico.cern.ch/event/804350/ contributions/3394378/attachments/1830532/2997717/ oeftiger_damper_instability.pdf.

[25] N. Mounet, Vlasov solvers and macroparticle simulations, in ICFA Mini-Workshop on Impedances and Beam Instabilities in Particle Accelerators, edited by V. Brancolini, G. Rumolo, M. Masullo, and S. Petracca (Benevento, Italy, 2017), p. 77 (https://doi.org/10.23732/CYRCP-2018001.77).

[26] A. Burov, Nested head-tail Vlasov solver, Phys. Rev. Accel. Beams 17, 021007 (2014). 